\title{
THE EFFECT OF COMPLETE AND PARTIAL CONTOUR ZIRCONIA COPINGS ON THE FRACTURE RESISTANCE OF ALL CERAMIC RESTORATIONS
}

Islam M Abdel Raheem, $M S c^{1}$,Sanaa $\mathrm{H}$ Abdel Kader, $P h D^{2}$, Fayza $\mathrm{H}$ Al Abbassy, $P h D^{3}$

\begin{abstract}
INTRODUCTION Veneer chipping is a common failure of zirconia based restorations, especially in the presence of high occlusal loads. Modifying zirconia design may influence veneer chipping.

OBJECTIVES: Purpose of this study was to evaluate fracture resistance of complete and partial contour zirconia crowns.

MATERIALS AND METHODS: Twenty standardized epoxy resin dies, prepared to receive zirconia based crowns, were divided into 4 groups, 5 specimens each. Zirconia framework designs were: (a) Full contour zirconia crown (FC), (b) Partial contour zirconia coping with buccal veneering (PC), (c) Anatomically reduced zirconia coping with palatal and proximal collar (ARD) and (d) half mm zirconia coping without collar (CON) (control group). Dual scanning technique was done for the test groups to fabricate zirconia copings with the use of standardized resin patterns, while normal scanning was made for the control group. After milling and sintering of zirconia specimens, veneering was done for all desired surfaces. All crowns were adhesively luted to their corresponding dies, and subjected to thermal cycling and mechanical loading corresponding to one year clinical service. Single load to fracture was applied to all the specimens. Type of failure was recorded for each specimen, investigated visually then by using Stereomicroscope and SEM.

RESULTS: Complete and partial contour zirconia restorations showed high resistance to fracture. The presence of collar was insignificant for increasing fracture resistance, but had an influence on the fracture extension toward the proximal surface of the veneered restorations.

KEYWORDS: Zirconia- Complete contour - Partial contour- Fracture resistance- Veneer chipping- Veneered zirconia.
\end{abstract}

1- Assistant lecturer of fixed prosthodontics, Conservative dentistry department, Alexandria University, Egypt

2- Professor of fixed prosthodontics, Conservative dentistry department, Alexandria University, Egypt.

3- Professor of dental materials, Dental Biomaterials Department, Alexandria University, Egypt.

\section{INTRODUCTION}

The use of zirconia for dental restorations has grown in recent years due to its high mechanical properties and relatively good esthetics. Yttria-stabilized tetragonal Zirconia polycrystals (Y-TZP) material formerly obtained opacious white color, making it only suitable to be used as a core material upon which esthetic veneering porcelain was applied, to enhance esthetic quality of the future restoration $(1,2)$.

The main clinical problem of bilayered zirconia restorations is the chipping of the weaker porcelain veneer, while the high strength zirconia core is mostly unaffected. Several factors have been discussed to reduce veneer chipping including modified coping designs, porcelain veneer materials with different fabrication techniques, modified veneer cooling protocol and matching thermal expansion coefficient of porcelain and zirconia (3).

Modified zirconia coping designs have been described in literature to reduce incidence of fracture. Bonfante et al (4) and Guess et al (5) found that anatomically reduced coping design, providing an even occlusal thickness of the veneer, showed higher reliability values than a constant thickness core. A similar design was investigated by Kokubo et al (6), and showed higher fracture load in vertical and lateral directions in comparison to a constant thickness coping design. A collared zirconia margin design of $2 \mathrm{~mm}$ height was recommended by Cho et al (7) and Liu et al (8), and showed higher fracture resistance than collarless coping designs.

Due to its relative translucency, newer formulations of (Y-TZP) have been introduced to be used in full contour configuration. Buer et al (9) and Preis et al (10) found full contour zirconia crown and FPD designs to obtain the highest fracture resistance in comparison to all types of veneered zirconia deigns or veneering materials. Although these formulations may present the ultimate solution to eliminate veneer chipping, they do not provide neither optical characteristics nor multiple range of shades and effects as do veneering porcelain materials; and cannot be applied in esthetically demanding situations(6).

$\mathrm{In}$ an attempt to minimize chipping of veneering porcelain in more esthetic areas, partial contour zirconia copings have been customized, in which porcelain veneered only the buccal aspect (11). It seems that this method may have advantages of both, veneered and full contour zirconia restorations; regarding the esthetic quality of veneered and the high strength of full contour zirconia. However, further researches are still needed to assess the effect of using full and partial contour zirconia designs to improve fracture resistance of zirconia crowns. The null hypothesis tested for this study was that modification of zirconia designs would not affect the fracture resistance of zirconia based restorations.

\section{MATERIALS AND METHODS}

A master metal die was fabricated using lost wax technique, to simulate maxillary first molar tooth prepared to receive all ceramic zirconia crown. The dimensions of the metal die was $6 \mathrm{~mm}$ height, $8 \mathrm{~mm}$ facio-lingually, $6 \mathrm{~mm}$ mesiodistally at the cervical level, with $1 \mathrm{~mm}$ circumferential chamfer finish line and 8 degrees axial walls taper. All transitions from the axial to the occlusal surfaces were made rounded and homogeneous. An acrylic resin hexagonal base was fabricated to help orientation of indices in later stages. 
Twenty epoxy resin dies $(\mathrm{N}=20)$ were fabricated by duplication of the master die using addition silicone duplicating material (Duosilim, SHERA WerkstoffTechnologie, Germany). These dies were divided into four groups $(n=5)$ representing different zirconia designs according to porcelain veneer extension: (1) Full contour zirconia (no veneer) FC, (2) Partial contour zirconia design (porcelain veneers only the buccal aspect) PC, (3) Anatomically reduced design with palatal and proximal collar (porcelain veneers all crown aspects except the collar area) ARD, (4) Conventional uniform thickness zirconia coping design (Porcelain veneers all crown aspects without collar) CON.

Full contour waxing was done on one epoxy resin die, simulating the dimensions of a maxillary left first molar (Fig.1, A); approximately $8 \mathrm{~mm}$ in crown height, $8 \mathrm{~mm}$ mesiodistally, and $10 \mathrm{~mm}$ faciolingually at the cervical level (12). The full contour waxed die was then duplicated into a stone die, which was set as a "reference die" to standardize contours of the final crowns (Fig. 1, B). For FC group, five condensation silicone putty and wash indices were fabricated to record external contours of the reference die, to fabricate 5 resin patterns which had the same shape and dimensions of the final restoration (Fig. 1, C).

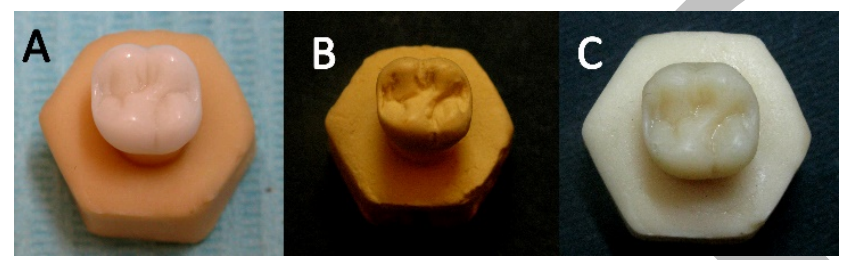

Fig (1): (A) Full contour waxing, (B) Reference stone die, (C) Resin pattern for FC group.

For PC group, $1.5 \mathrm{~mm}$ cutback was performed at the buccal surface and buccal one third of the occlusal surface of the same full contour waxed die which was duplicated into a stone die as done before for the previous group (Fig. 2, A and B). This was followed by fabrication of five resin patterns as performed earlier. For ARD group, $1.5 \mathrm{~mm}$ wax cutback was extended to all crown aspects of the same waxed die, leaving a proximal and palatal collar, followed by the same procedures till obtaining the 5 resin patterns of this group (Fig. 2, C and D). While for CON group, no resin patterns were fabricated, and the design of the zirconia copings was determined by software settings of the CAD software.

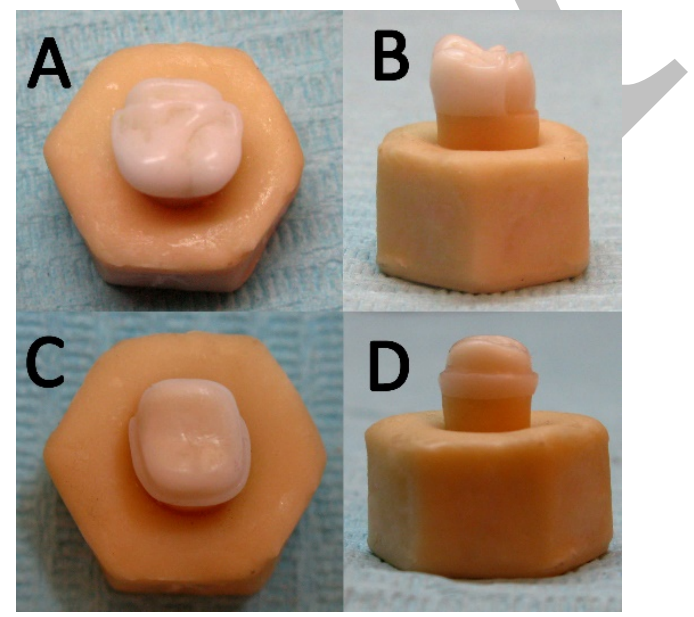

Fig (2): Wax cut back for PC group (A and B) and ARD group (C and D).
All dies of FC, PC and ARD groups were scanned (S600 3D optical scanner, Zirkonzahn, Italy) using dual scan technique, in which two scans for the same die were performed with and without the resin pattern. After scanning was completed, finish line of the scanned dies, and the margins of the resin pattern in the die-pattern scanning were identified by the CAD software (Zirkonzahn Modellier, Zirkonzahn, Italy). Each resin pattern was virtually adapted onto its corresponding die by matching the margins to the finish line. On contrary, normal scanning was performed for the dies of CON group, and the design of zirconia coping was determined completely by CAD software, which was set as $0.5 \mathrm{~mm}$ uniform thickness coping as mentioned earlier.

All zirconia copings were manufactured by subtractive milling of pre-sintered green state zirconia blanks (Prettau Zirconia, Zirkonzahn, Italy). After milling was completed, zirconia was sintered in a twelve-hour-cycle at $1600{ }^{\circ} \mathrm{C}$ using zirconia sintering furnace (Zirkofen 600, Zirkonzahn, Italy). Specimens were then checked for seating onto their corresponding dies. All zirconia framworks are shown in (Fig. 3). Veneering of specimens was done for groups PC, $\mathrm{ARD}$ and $\mathrm{CON}$ using dental porcelain veneering material (Ice Zirconia Ceramic, Zirkonzahn, Italy). All contours of veneered restorations were controlled using putty indices taken from the reference stone die. All specimens were cemented using dual cure resin cement (Relyx ARC, 3MESPE, USA), under $5 \mathrm{Kg}$ static load for $10 \mathrm{~min}(13,14)$.

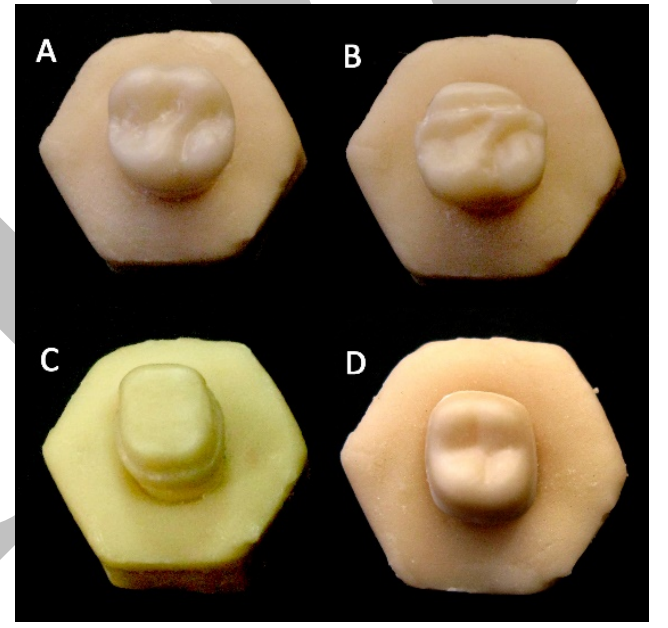

Fig (3): Zirconia designs of all groups; (A) FC, (B) PC, (C) $\mathrm{ARD}$, and (D) CON.

All the specimens were subjected to thermocycling and mechanical loading (TCML) (dental biomaterial laboratories, faculty of dentistry, Alexandria University) corresponding to one year clinical service (15). Specimens were first subjected to 1200 thermal cycles between $5-55^{\circ} \mathrm{C}$ in a two min. cycle. Mechanical loading was done using 6 mm sphere indenters carrying $5 \mathrm{Kg}$ load, with a speed of 2 HZ for 240000 cycles. All specimens were then subjected to load-to-fracture testing using universal testing machine (AGS-X 100KN, Shimadzu, Japan). An eight mm diameter stainless steel sphere was used for this purpose. Every effort was made to make sure that the sphere would touch the occlusal surface of each specimen in three points; occlusal inclines of the mesiobuccal, distobuccal and mesiopalatal cusps of each crown. A piece of polyethylene sheet was placed between the sphere and the specimen, in order to properly distribute the load. A crosshead speed of $1 \mathrm{~mm} / \mathrm{min}$ 
was chosen. After starting the operation, the load was raised gradually, until sudden sharp decrease of the force, which was also accompanied by failure of the specimens either in the veneer only or combined core/ veneer failure. The maximum load before the sharp decrease of force was recognized as the "fracture load", and was determined for each specimen in newton.

Stereomicroscope was used to classify mode of failure of each specimen into either veneer only fracture or combined veneer and core fracture. Further qualitative evaluation was done using scanning electron microscope.

Statistical analysis:

Data were analyzed using statistical software (IBM SPSS software package version 20.0). Quantitative data were described using range (minimum and maximum), mean, standard deviation and median. Comparison between the four groups were done using Kruskal Wallis test and pair wise comparison was assessed using Mann-Whitney test. Significance of the obtained results was judged at the $5 \%$ level.

\section{RESULTS}

For fracture resistance test, the highest mean fracture load of all groups was that of FC group $(6104.4 \pm 992.2 \mathrm{~N})$. PC was the second, and had mean fracture load of (4263.8 \pm $867.9 \mathrm{~N})$, then ARD group $(2950.4 \pm 705.9 \mathrm{~N})$, and the lowest mean was recorded in CON group $(2625.3 \pm 915.08$ $\mathrm{N})$. All these data are presented in table (1) and graph (1).

\section{Means of Fracture Loads with Standard Deviation Error Bars}

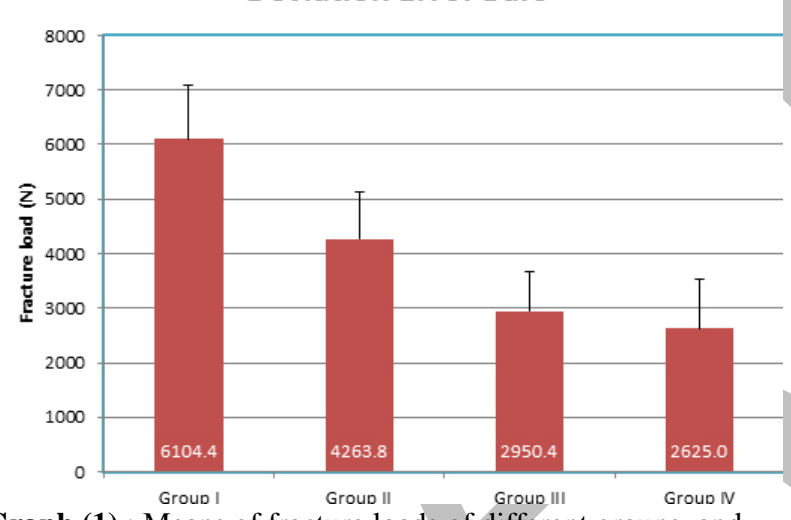

Graph (1) : Means of fracture loads of different groups, and standard deviation error bars.

Table (1) Comparison between the different groups according to Fracture Load

\begin{tabular}{|c|c|c|c|c|c|c|}
\hline & $\begin{array}{c}\text { Group I } \\
(n=5)\end{array}$ & $\begin{array}{c}\text { Group II } \\
(\mathrm{n}=5)\end{array}$ & $\begin{array}{c}\text { Group III } \\
(n=5)\end{array}$ & $\begin{array}{c}\text { Group IV } \\
(\mathrm{n}=5)\end{array}$ & ${ }^{\mathrm{KW}} \chi^{2}$ & $\mathbf{p}$ \\
\hline $\begin{array}{l}\text { Fracture } \\
\text { Load (N) }\end{array}$ & & & & & \multirow{4}{*}{$\begin{array}{c}14.55 \\
4^{*}\end{array}$} & \multirow{4}{*}{$\begin{array}{c}0.00 \\
2^{*}\end{array}$} \\
\hline $\begin{array}{l}\text { Min - } \\
\text { Max }\end{array}$ & $\begin{array}{c}4881.3- \\
7637.5\end{array}$ & $\begin{array}{c}3415.6- \\
5631.3\end{array}$ & $\begin{array}{c}1745.1- \\
3527.9\end{array}$ & $\begin{array}{c}1493.8- \\
3562.3\end{array}$ & & \\
\hline $\begin{array}{l}\text { Mean } \\
\pm \text { SD. }\end{array}$ & $\begin{array}{c}6104.4 \pm \\
992.2\end{array}$ & $\begin{array}{c}4263.8 \pm \\
867.9\end{array}$ & $\begin{array}{c}2950.4 \pm \\
705.9\end{array}$ & $\begin{array}{l}2625 \pm \\
915.08\end{array}$ & & \\
\hline $\begin{array}{l}\text { Media } \\
\mathrm{n}\end{array}$ & 5996.88 & 4281.25 & 3078.13 & 2509.38 & & \\
\hline $\mathbf{p}_{1}$ & & \multirow[t]{3}{*}{$0.016^{*}$} & $0.009^{*}$ & $0.016^{*}$ & & \\
\hline $\mathbf{p}_{2}$ & & & $0.016^{*}$ & $0.028^{*}$ & & \\
\hline $\mathbf{p}_{3}$ & & & & 0.917 & & \\
\hline
\end{tabular}

Kruskal-Wallis statistical test revealed a significant difference between the parallel groups $(p=0.002)$. Mann Whitney test also revealed significant differences between two-group sets. FC group has the highest statistically significant fracture load than the other three groups, while PC group was significantly higher in fracture load than ARD and CON group. There was no statistical significant difference between the two later groups.

Evaluation of the fractured specimens visually and using stereomicroscope was performed to classify each specimen into a failure mode; veneer only fracture and combined veneer and core fracture (16).

For FC group, all crowns were fractured into multiple pieces, detached from corresponding dies that were fractured or cracked as well. Because specimens of this group were monolithic zirconia, failure mode cannot be compared with the other groups. PC group had one specimen with veneer fracture (20\%) shown in (fig. 4, C, D and $\mathrm{E}$ ), while the other four specimens (80\%) were fractured at both core and veneer. Two of the specimens with the combined fracture failed into multiple pieces. One specimen failed by crack in the core accompanied by chipping of the veneer (fig. 4, A and B). The last one showed a crack in the core beneath a chipped veneer area, and a chipped zirconia piece at palatal aspect of the crowns.

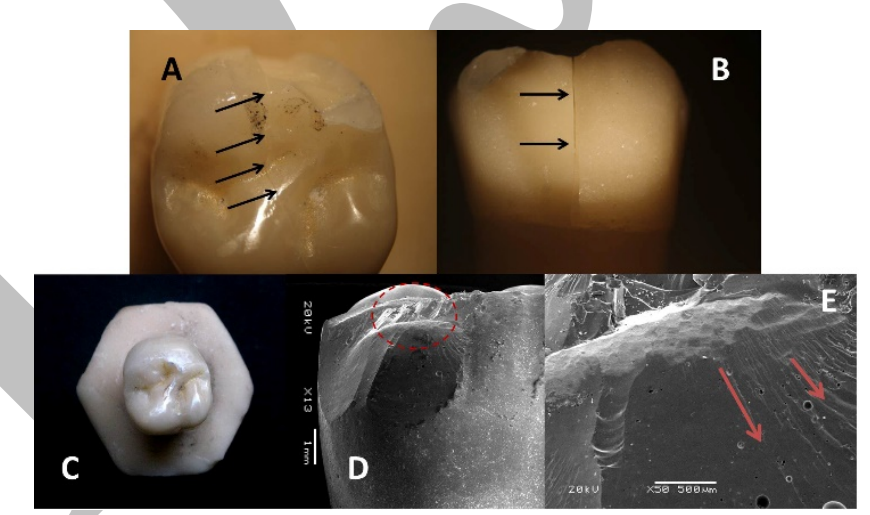

Fig. (4) PC Group, (A and B) Stereomicroscope images showing combined core and veneer fracture. Black arrows points at a crack line in the zirconia surface (closed fracture). (C, D and E) Veneer only fracture; (C) digital image of the chipped specimen, (D, E) Scanning electron micrographs of the same specimen, dashed circle denotes the fracture origin, arrows show hackles and wake hackles, indicating the direction of crack propagation.

For ARD group, veneer fracture occurred in 3 specimens (60\%) with an area nearly one quarter of the crown at buccal surface and extending no more than half of the proximal surface, while two specimens (40\%) showed combined fracture into multiple pieces. CON group had veneer fracture in two specimens (40\%) with one specimen had chipping area extending more than one quarter of the crown, toward the proximal surface. Combined veneer and core fracture in CON group occurred in 3 specimens (60\%); one specimen had a small detached fragment of both veneer and core while the other 2 specimens were fractured into multiple pieces.

SEM and stereomicroscope images revealed origin of fracture in representative specimens in all groups. In FC group, origin of fracture was either form occlusal surface (Hertzian cone crack) (fig. 5), or from intaglio of the crown (radial crack). Also compression curls were seen in more 
than one specimen. In the specimens examined of the three other groups, specimens failed by cone cracking, with hackles and wake hackles pointing toward the core (direction of crack propagation toward the core) (fig. $5 \mathrm{D}$ and $\mathrm{E})$.

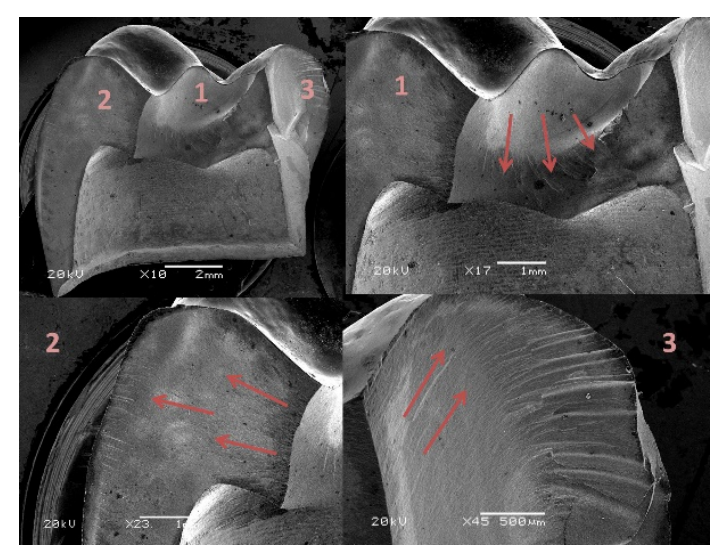

Fig. (5) SEM images of a crown fragment in FC group, (1) Fracture origin (Hertzian cone crack). (2) Hackles denote the direction of crack propagation. (3) 2 fractographic features are apparent; hackles and compression curls.

\section{DISCUSSION}

Translucent zirconia materials have been increasingly used in monolithic full contour configuration to restore posterior teeth, to overcome chipping problem associated with veneered zirconia restorations. Veneering of such material can be done for areas in the mouth with high esthetic requirements, but this is accompanied with the possibility of veneer chipping. Modifying zirconia framework design was considered in this study as a factor to eliminate chipping.

Selection of the framework designs for the test groups was based on observation of clinical reports $(11,17,18)$, and other in vitro studies $(4-6,13,19,20)$ of zirconia based restorations. Full contour zirconia restorations are promising, but yet not desirable in high esthetic areas. As a result, in this current study, modification of zirconia framework was made in two designs; the first was to veneer only the buccal surface, with lapping of the buccal cusps, and the second was to anatomically design a zirconia framework to support an even thickness of veneer, with palatal and proximal collar to support the veneer.

Marchack et al (11) were the first to describe complete and partial contour zirconia restorations. PC Group in the current study resembled the partial contour restorations described in the previously mentioned study, which in turn resembled what was previously proposed for porcelain fused to metal crowns (21). For ARD group, collar of $2 \mathrm{~mm}$ height was designed at the palatal and proximal surface to support the veneer. Many reports of chipped porcelain at the proximal surface gave a reason for extending the collar proximally as done in this study $(17,18,22)$. However, conventional coping design in CON group was made with even thickness of $0.5 \mathrm{~mm}$, to serve as a control group.

Cementation protocol was standardized for all specimens, using the same resin cement. Although cementation protocol was of little concern for single load to fracture testing, standardization was very important to achieve, in order not to change the elastic modulus underneath the crown specimen (23).

Although some all ceramic materials exhibited same fracture loads with or without thermocycling and mechanical loading (TCML), zirconia material is affected by aging due to low temperature degradation phenomenon (24). Thermal cycling regime was conducted to simulate intraoral temperature changes, and aging of the restorations using repeated applications of chewing forces to study the effect of cyclic loading on the strength of test groups during service.

During the cyclic loading, sphere indenters with a diameter of $6 \mathrm{~mm}$ were used as antagonists in this study according to previous investigations done by Zou et al (25), Beuer et al (26) and Albrecht et al (27).

Fracture testing was done using sphere indenter with a diameter of $8 \mathrm{~mm}$ to ensure 3 point contacts with the occlusal surface of the specimen (28). This was of high importance for PC group, where veneering porcelain extended to the buccal half of the buccal cusps. One mm polyethylene sheet was used to interpose the contact between the ball and the specimen, in order to further distribute the force as recommended by Kelly (29). In the latter review, it was also recommended to use large diameter loading pistons, but application of such large diameters would result in extremely high fracture loads, especially when testing high strength ceramic such as zirconia. In a study conducted by Beuer et al (9), some of the tested full contour zirconia crowns resisted fracture when subjected to the highest force that can be applied to universal testing machine used (10500 N). Authors referred the high loads to the large diameter piston $(10 \mathrm{~mm})$.

From the given results of the current study, the null hypothesis was rejected. It was obvious that full contour zirconia group had statistically significant superior fracture load (6104.4 $\pm 992.2 \mathrm{~N})$. This is absolutely referred to the monolithic design of the zirconia crowns, without weak veneering porcelain. This result was in agreement with the study conducted by Beuer et al (2012) (9), in which a significant difference in fracture resistance was recorded between full contour zirconia crowns and conventionally veneered zirconia crowns.

PC group showed high fracture load (4263.8 $\pm 867.9 \mathrm{~N})$, and was significantly higher than ARD (2950.4 \pm 705.9$)$ and CON (2645.0 $\pm 940.6 \mathrm{~N})$ groups. The high mean fracture load of ARD group might be because this group was partially veneered, so that the sphere indenter had contacts with both zirconia surface at the palatal cusp and veneering porcelain at the buccal cusps. There are no enough studies testing posterior partially veneered zirconia coping presented in group II, except for one finite element analysis study, in which Porojan et al (30) found that the buccal veneered design had the least maximal principal stress at the veneer, and the highest at zirconia core material. Also, clinical case series performed by Marchack et al (11) assumed that there was no chipping occurred after one year follow up.

ARD group showed no statistical difference from CON group, this may be due to the anatomical preparation of abutment teeth which made the $0.5 \mathrm{~mm}$ thickness conventional coping of CON group partially supporting the veneer.

In literature, there are many studies that have been done to compare modified and standard coping designs, but with different modifications of the framework and different failure testing methods. Modifications in framework design included collared designs $(8,13,31-33)$, anatomical cut back designs $(5,19,34)$, and also included addition of ribs or 
horizontal embossments $(35,36)$. Testing methods included single load to fracture $(8,20,31)$, reliability and survival rate after fatigue testing $(4,5,13,14)$, measuring the number and total area of chipping (37) and Finite Element Analysis (FEA) (36). Added to the previous differences, numerous zirconia and veneering ceramic materials were used in these studies and are available in the market. These factors might lead to the diversity of results.

Results of the current study were in agreement with the results of a study conducted by Lorenzoni et al (13) in which there was no statistical significant difference between collared and collarless designs, where the collar extended at the lingual and proximal margins. In the same issue, Kokubo et al (6) found that placing a collar had no significant effect upon increasing fracture load when subjected to vertical loading.

On contrary, these results were in disagreement with those of studies conducted by Bonfante et al (4), Silva et al (19) and Guess et al (5). These studies were almost in the same direction, with little differences in between but the same results. Authors of these studies found collared anatomically reduced designs more reliable than standard copings. This difference may be because these studies tested the reliability of the zirconia crowns after fatigue testing, while fracture resistance was tested in this study.

Although Burke and Watts (38) classification has been used in literature to evaluate failure mode, it could not describe the mode of fracture of either the high strength monolithic zirconia crowns as in FC group, or bilayered veneered zirconia restorations as in PC, ARD and CON groups. Reasons could be due to the fact that monolithic zirconia had different behavior of fracture from the glass ceramic crowns. Zirconia crowns tend to have catastrophic fracture into multiple fragment pieces, and hence cannot be classified into any mode but catastrophic failure. Secondly, in bilayered restorations such as veneered zirconia restorations, fracture of the veneer might occur alone, or accompanied by core fracture.

In this current study, for PC group, although the $8 \mathrm{~mm}$ sphere indenter was ensured to make contact with both veneering porcelain and zirconia, combined fractures occurred more frequently than veneer only fractures. As mentioned earlier, this may be because the sphere indenter had contacts at both zirconia and veneering porcelain.

From stereomicroscope images, in ARD group collared anatomical coping design had smaller chipping fragments than do CON group. The chipping did not extend to the proximal surface where collar is present. In the opposite, chipping in CON group extended toward the proximal surface in one specimen. The later result is in agreement with clinical reports of proximal areas of the veneer chipped from zirconia restorations that were replaced with new crowns with collared proximal margins $(11,18,22)$.

SEM images of the examined specimens in this current study revealed origins of fracture to be either from the occlusal surface (cone cracks), or from the intaglio surface of the crown (radial cracking). In FC group, both origins were identified, and hackles were seen, and aided the identification of the crack path. Compression curls (cantilever curls) were also identified, which suggested the presence of high stresses at fracture.

For PC, ARD and CON groups, it was more difficult to identify origins of fracture, as there were 2 substrates. For the examined specimens, all specimens that showed veneer only fracture failed with cone cracks at the occlusal surfaces. Hackles and wake hackles were traced to be sure of the cause of failure, and to indicate direction of crack propagation. Arrest lines were also seen, and its convex surface indicated direction of crack propagation. These results are in agreement with Rosentritt et al (37), Lorenzoni et al (13) and Cho et al (39). Also these results shows similarity to results of fractographic analysis done to investigate fractured fragments retrieved from clinically failed crowns $(22,40)$

Complete and partial contour zirconia restorations showed high resistance to fracture, and offered ease of fabrication, with less time and less veneering material used for the partial contour zirconia design, in comparison to other veneered zirconia restorations. Partial contour zirconia design offers an esthetic alternative to the complete contour design. Partial contour zirconia design can also be recommended to function as a retainer for fixed partial denture, with the benefit of having full diameter of the connector made of zirconia without veneering.

\section{CONCLUSION}

Within limitations of this study, the following conclusions can be drawn:

All presented framework designs, including the control group, showed potential to withstand physiologic occlusal forces applied in the posterior region.

Complete contour zirconia crowns showed the highest statistically significant mean of fracture resistance.

Partial contour zirconia design showed a statistically significant higher mean of fracture load than the collared and non-collared groups.

Presence of a collar had no effect on the fracture resistance, but had an influence on the fracture extension toward the proximal surface of the veneered zirconia restorations.

\section{CONFLICT OF INTEREST}

The authors declare that they have no conflicts of interest.

\section{ACKNOWLEDGEMENT}

We would like to thank Mr. Sherif El Dabaa for his assistance in the laboratory phase of the study.

\section{REFERENCES}

1. Guazzato M, Albakry M, Ringer SP, Swain M V. Strength, fracture toughness and microstructure of a selection of allceramic materials. Part II. Zirconia-based dental ceramics. Dent Mater. 2004 ;20:449-56.

2. Vichi A, Louca C, Corciolani G, Ferrari M. Color related to ceramic and zirconia restorations: A review. Dent Mater. 2011;27:97-108.

3. Raigrodski AJ, Hillstead MB, Meng GK, Chung K-H. Survival and complications of zirconia-based fixed dental prostheses: A systematic review. J Prosthet Dent. 2012 ;107:170-7.

4. Bonfante EA, Zavanelli RA, Silva NRFA, Elizabeth D, Thompson VP, Ea B, et al. Thermal / mechanical simulation and laboratory fatigue testing of an alternative yttria tetragonal zirconia layered crown design. Eur J Oral Sci. 2010;118: 202-9.

5. Guess PC, Bonfante EA, Silva NRFA, Coelho PG, Thompson VP. Effect of core design and veneering 
technique on damage and reliability of Y-TZP-supported crowns. Dent Mater. 2013;29:307-16.

6. Kokubo Y, Tsumita M, Kano T, Fukushima S. The influence of zirconia coping designs on the fracture load of all-ceramic molar crowns. Dent Mater J. 2011;30:281-5.

7. Jang GW, Kim HS, Choe HC, Son MK. Fracture strength and mechanism of dental ceramic crown with zirconia thickness. Procedia Eng. 2011;10:1556-60.

8. Liu Y, Wang Y, Zhang Q, Gao Y, Feng H. Fracture reliability of zirconia all-ceramic crown according to zirconia coping design. Beijing Da Xue Xue Bao. 2014 Feb 18;46:71-5.

9. Beuer F, Stimmelmayr M, Gueth JF, Edelhoff D, Naumann M. In vitro performance of full-contour zirconia single crowns. Dent Mater. 2012;28:449-56.

10. Preis V, Behr M, Hahnel S, Handel G, Rosentritt M. In vitro failure and fracture resistance of veneered and full-contour zirconia restorations. J Dent. 2012;40:921-8.

11. Marchack BW, Sato S, Marchack CB, White SN. Complete and partial contour zirconia designs for crowns and fixed dental prostheses: A clinical report. J Prosthet Dent . 2011;106:145-52.

12. Nelson SJ, Ash MM, Ash MM. Wheeler's dental anatomy, physiology, and occlusion. Saunders/Elsevier; 2010. 346 p.

13. Lorenzoni FC, Martins LM, Silva NRFA, Coelho PG, Guess PC, Bonfante EA, et al. Fatigue life and failure modes of crowns systems with a modified framework design. J Dent. 2010;38:626-34.

14. Kim JH, Park JH, Park YB, Moon HS. Fracture load of zirconia crowns according to the thickness and marginal design of coping. J Prosthet Dent. 2012;108:96-101.

15. Rosentritt M, Behr M, van der Zel JM, Feilzer AJ. Approach for valuating the influence of laboratory simulation. Dent Mater. 2009;25:348-52.

16. Stawarczyk B, Ozcan M, Hammerle CHF, Roos M. The fracture load and failure types of veneered anterior zirconia crowns: An analysis of normal and Weibull distribution of complete and censored data. Dent Mater. 2012;28:478-87.

17. Marchack BW, Futatsuki Y, Marchack CB, White SN. Customization of milled zirconia copings for all-ceramic crowns: A clinical report. J Prosthet Dent. 2008;99:169-73.

18. Pogoncheff CM, Duff RE. Use of zirconia collar to prevent interproximal porcelain fracture: A clinical report. J Prosthet Dent. 2010;104:77-9.

19. Silva NRFA, Bonfante EA, Rafferty BT, Zavanelli RA, Rekow ED, Thompson VP, et al. Modified Y-TZP Core Design Improves All-ceramic Crown Reliability. J Dent Res. 2011;90:104-8.

20. Amir Rad FA, Succaria FG, Morgano SM. Fracture resistance of porcelain veneered zirconia crowns with exposed lingual zirconia for anterior teeth after thermal cycling: An in vitro study. Saudi Dent J. 2015;27:63-9.

21. Rosenstiel SF, Land MF, Fujimoto J. Contemporary fixed prosthodontics. $879 \mathrm{p}$.

22. Scherrer SS, Quinn JB, Quinn GD, Wiskott HWA. Fractographic ceramic failure analysis using the replica technique. Dent Mater . 2007;23:1397-404.

23. Rekow E, Harsono M, Janal M, Thompson V, Zhang G. Factorial analysis of variables influencing stress in allceramic crowns. Dent Mater. 2006 Feb;22:125-32.

24. Lughi V, Sergo V. Low temperature degradation -aging- of zirconia: A critical review of the relevant aspects in dentistry. Dent Mater. 2010;26:807-20.
25. Zou L, Samarawickrama D, Seymour K, Stout K. Free Form Surface Measurement Using Non-Contact Measurement Methodology. XVII IMEKO World congress, Dubrovnik, Croatia. 2003;1882-5.

26. Beuer F, Steff B, Naumann M, Sorensen JA. Load-bearing capacity of all-ceramic three-unit fixed partial dentures with different computer-aided design (CAD)/computer-aided manufacturing (CAM) fabricated framework materials. Eur J Oral Sci. 2008;116:381-6.

27. Albrecht T, Kirsten A, Kappert HF, Fischer H. Fracture load of different crown systems on zirconia implant abutments. Dent Mater. 2011; 27:298-303.

28. Heintze SD, Cavalleri A, Zellweger G, Büchler A, Zappini G. Fracture frequency of all-ceramic crowns during dynamic loading in a chewing simulator using different loading and luting protocols. Dent Mater. 2008;24:135261.

29. Kelly JR. Clinically relevant approach to failure testing of all-ceramic restorations. J Prosthet Dent. 1999;81:652-61.

30. Porojan L, Topala F, Porojan S, Savencu C. Effect of frame design and veneering material on biomechanical behavior of zirconia dental crowns veneered with overpressing ceramics. Dent Mater J. 2017;36:275-81.

31. Sundh A, Sjögren G. A comparison of fracture strength of yttrium-oxide-partially-stabilized zirconia ceramic crowns with varying core thickness, shapes and veneer ceramics. J Oral Rehabil. 2004;31:682-8.

32. Okabayashi S, Nomoto S, Sato T, Miho O. Influence of proximal supportive design of zirconia framework on fracture load of veneering porcelain. Dent Mater $\mathrm{J}$. 2013;32:572-7.

33. Cho HY, Won HY, Choe HC, Son MK. Fracture characteristics of dental ceramic crown according to zirconia coping design. Procedia Eng. 2011;10:1561-6.

34. Silva NRFA, Bonfante EA, Zavanelli RA, Thompson VP, Ferencz JL, Coelho PG. Reliability of Metalloceramic and Zirconia-based Ceramic Crowns. J Dent Res. 2010;89:1051-6.

35. Rosentritt M. A focus on zirconia : an in-vitro lifetime prediction of zirconia dental restorations. University of Amesterdam; 2008.

36. Sung M, Han CH, Kim S. A novel coping design to decrease maximum principal stress in zirconia ceramic restorations. J Prosthodont. 2014;23:534-9.

37. Rosentritt M, Steiger D, Behr M, Handel G, Kolbeck C. Influence of substructure design and spacer settings on the in vitro performance of molar zirconia crowns. J Dent. 2009;37:978-83.

38. Burke FJ, Watts DC. Fracture resistance of teeth restored with dentin-bonded crowns. Quintessence Int . 1994 ;25:335-40.

39. Cho HY, Won HY, Choe HC, Son MK. Fracture characteristics of dental ceramic crown according to zirconia coping design. Procedia Eng. 2011;10:1561-6.

40. Oilo $M$, Hardang AD, Ulsund AH, Gjerdet NR. Fractographic features of glass-ceramic and zirconia-based dental restorations fractured during clinical function. Eur $\mathrm{J}$ Oral Sci. 2014;122:238-44. 